\title{
Testing different brain metastasis grading systems in stereotactic radiosurgery: Radiation Therapy Oncology Group's RPA, SIR, BSBM, GPA, and modified RPA
}

\author{
Clinical article
}

\author{
Toru Serizawa, M.D., ${ }^{1}$ Yoshinori Higuchi, M.D., ${ }^{2}$ Osamu Nagano, M.D. ${ }^{3}$ Tatsuo Hirai, M.D.,${ }^{4}$ \\ Junichi Ono, M.D., ${ }^{3}$ Naokatsu Saeki, M.D., ${ }^{2}$ and Akifumi Miyakawa, M.D. ${ }^{4,5}$ \\ ${ }^{1}$ Tokyo Gamma Unit Center, Tsukiji Neurologic Clinic, Tokyo; ${ }^{2}$ Department of Neurological Surgery, Chiba University \\ Graduate School of Medicine, Chiba; ${ }^{3}$ Gamma Knife House, Chiba Cardiovascular Center, Ichihara; ${ }^{4}$ Stereotaxis and \\ Gamma Unit Center, Fujieda Heisei Memorial Hospital, Fujieda; and ${ }^{5}$ Department of Radiology, Graduate School of \\ Medical Science and Medical School, Nagoya City University, Nagoya, Japan
}

\begin{abstract}
Object. The authors conducted validity testing of the 5 major reported indices for radiosurgically treated brain metastases the original Radiation Therapy Oncology Group's Recursive Partitioning Analysis (RPA), the Score Index for Radiosurgery in Brain Metastases (SIR), the Basic Score for Brain Metastases (BSBM), the Graded Prognostic Assessment (GPA), and the subclassification of RPA Class II proposed by Yamamoto-in nearly 2500 cases treated with Gamma Knife surgery (GKS), focusing on the preservation of neurological function as well as the traditional endpoint of overall survival.

Methods. The authors analyzed data from 2445 cases treated with GKS by the first author (T.S.), the primary surgeon. The patient group consisted of 1716 patients treated between January 1998 and March 2008 (the Chiba series) and 729 patients treated between April 2008 and December 2011 (the Tokyo series). The interval from the date of GKS until the date of the patient's death (overall survival) and impaired activities of daily living (qualitative survival) were calculated using the Kaplan-Meier method, while the absolute risk for two adjacent classes of each grading system and both hazard ratios and $95 \%$ confidence intervals were estimated using the Cox proportional hazards model.

Results. For overall survival, there were highly statistically significant differences between each two adjacent patient groups characterized by class or score (all p values $<0.001$ ), except for GPA Scores 3.5-4.0 and 3.0. The SIR showed the best statistical results for predicting preservation of neurological function. Although no other grading systems yielded statistically significant differences in qualitative survival, the BSBM and the modified RPA appeared to be better than the original RPA and GPA.

Conclusions. The modified RPA subclassification, proposed by Yamamoto, is well balanced in scoring simplicity with respect to case number distribution and statistical results for overall survival. However, a new or revised grading system is necessary for predicting qualitative survival and for selecting the optimal treatment for patients with brain metastasis treated by GKS. (http://thejns.org/doi/abs/10.3171/2012.8.GKS12710)
\end{abstract}

$\begin{array}{ll}\text { KEY WORDS } & \text { brain metastasis } \bullet \quad \text { overall survival } \bullet \quad \text { grading system } \\ \text { qualitative survival } & \bullet \quad \text { Gamma Knife surgery } \bullet \quad \text { stereotactic radiosurgery }\end{array}$

I N 2012, Yamamoto et al. ${ }^{16}$ proposed a new subclassification for Class II patients in the Recursive Partitioning Analysis (RPA), which was originally reported by Gaspar et al. ${ }^{3}$ in 1997 . This new index was relatively simple to use in a clinical setting and proved to be applicable to patients with brain metastases who were

\footnotetext{
Abbreviations used in this paper: BSBM = Basic Score for Brain Metastases; GKS = Gamma Knife surgery; GPA = Graded Prognostic Assessment; KPS = Karnofsky Performance Scale; RPA = Recursive Partitioning Analysis; SIR = Score Index for Radiosurgery in Brain Metastases; SRS = stereotactic radiosurgery; $\mathrm{SRT}=$ stereotactic radiotherapy; $\mathrm{WBRT}=$ whole-brain radiation therapy.
}

treated with SRS. Several grading systems are available, however, such as the Score Index for Radiosurgery in Brain Metastases (SIR, Weltman and colleagues, 2000), ${ }^{14}$ the Basic Score for Brain Metastases (BSBM, Lorenzoni and coworkers, 2000), ${ }^{7}$ and the Graded Prognostic Assessment (GPA, Sperduto et al., 2008). ${ }^{13}$ These systems are all used for predicting overall survival alone and do not apply to preservation of neurological function. In the current study, we tested the validity of the 5 major indices - the original RPA, SIR, BSBM, GPA, and the modified RPA - in nearly 2500 cases treated with GKS, focusing on preservation of neurological function as well as the traditional endpoint of overall survival. 


\section{Methods}

We analyzed data in 2445 cases after we had obtained approval from our local institutional review board. The database contained information from two patient cohorts studied retrospectively: the Chiba series, composed of 1716 consecutively enrolled patients treated between January 1998 and March 2008; and the Tokyo series, composed of 729 patients treated between April 2008 and December 2011. All aspects of patient selection, radiation dose planning, dose selection, performance of GKS, and collection of follow-up data were undertaken by the first author (T.S.). During the 14-year period from 1998 to 2011, all these patients were treated according to the same protocol, as reported previously. ${ }^{8-11}$ At the initial treatment, all lesions were irradiated by SRS without upfront WBRT. In some cases in which tumor volumes exceeded $10 \mathrm{~cm}^{3}$, staged SRT was chosen ${ }^{4}$; and in all cases in which the total tumor volume exceeded $20 \mathrm{~cm}^{3}$ and/or the number of tumors was greater than 25 , the radiosurgical procedure was divided into 2 or 3 sessions to ensure a total skull integral dose of less than $10 \mathrm{~J}$, thereby preventing acute brain swelling. ${ }^{11,15}$ New distant lesions, detected by Gd-enhanced MR imaging, which was performed every 2-3 months, were treated mainly with GKS, although if cerebral or CSF tumor dissemination was detected, these lesions were treated with WBRT. The treatment strategy was explained in detail to each patient, and written informed consent was obtained from all patients before GKS.

After the patient had received relatively deep sedation and a local anesthetic agent had been applied to the patient's head, a stereotactic coordinate frame (Leksell model G stereotactic coordinate frame, Elekta AB) was affixed to the head. For target coordinate determination and dose-planning, stereotactic Gd-enhanced T1weighted axial MR images (slice thickness $2 \mathrm{~mm}$, multiple slices) covering the entire brain were obtained. For dose planning, we used the Leksell GammaPlan (Elekta). We used a Leksell Gamma Knife (Elekta) for all procedures: before October 2003 (1988-2003), we performed GKS using model B; from October 2003 to November 2011, we used model C; and from December 2001 onward, we used the model Perfexion. The standard prescribed dose directed to the tumor periphery, 18-24 Gy, was changed depending on the pathological characteristics of the tumor, the patient's physical status, the tumor location and volume, the status of the patient's extracranial disease, and so forth.

All data were analyzed according to the intention-totreat principle. The intervals from the date of GKS until the date of death (overall survival) and impaired activities of daily living (qualitative survival) were calculated using the Kaplan-Meier method. ${ }^{5}$ The estimated absolute risk for two adjacent classes of each grading system, as well as hazard ratios and $95 \%$ confidence intervals, were calculated using the Cox proportional hazards model. ${ }^{2}$ Prognostic values for overall survival were also calculated using the Cox proportional hazard model, which considered 14 factors: patient age and sex, initial KPS score, primary site of the lesion, number of tumors, total tumor volume, MR imaging findings of CSF dissemination, craniotomy, chemotherapy, lag between diagnosis of prima- ry cancer and brain metastasis, prior WBRT, maximum tumor diameter, neurological symptoms, and status of the extracranial disease. Impaired activities of daily living were defined as an impaired neurological status that is reflected by a KPS score $6<70 \%$, as reported by Aoyama et al. ${ }^{1}$ Thus, cases without improvement to KPS scores of $\geq 70 \% 3$, even after GKS, were excluded when evaluating the qualitative survival. The differences between two adjacent classes were compared using the log-rank test. A p value less than 0.01 was defined as statistically significant. All statistical analyses were performed using the JMP software program, version 9.0.3 (SAS Institute Inc.).

\section{Results}

At the end of December 2011, 353 patients (14.4\%) were still alive, and the remaining 2092 patients (85.6\%) were confirmed to have died. Table 1 summarizes the demographic characteristics of the patients. The median overall survival time was 7.8 months. The cumulative overall survival rates were $60.0 \%$ at 6 months, $35.4 \%$ at 12 months, $15.8 \%$ at 24 months, $9.4 \%$ at 36 months, $4.0 \%$ at 60 months, and $0.1 \%$ at 120 months after GKS. The cumulative qualitative survival rates were $90.5 \%$ at 6 months, $80.3 \%$ at 12 months, $64.3 \%$ at 24 months, $56.0 \%$ at 36 months, $43.2 \%$ at 60 months, and $23.3 \%$ at 120 months after the procedure. Among the 2092 deceased patients, the causes of death were determined to be brain diseases in 298 patients (14.2\%) and systemic diseases in 1794 patents (85.8\%). Salvage repeated GKS procedures for new distant and/or recurrent brain metastases after the initial GKS were required in 1031 patients $(42.2 \%)$ and salvage WBRT in 95 patients (3.9\%).

Figures 1-5 demonstrate overall survival and qualitative survival curves according to the 5 major grading systems (Fig. 1, RPA; Fig. 2, SIR; Fig. 3, BSBM; Fig. 4, GPA; and Fig. 5, the modified RPA). Statistical results for overall survival are presented in detail in Tables 2 and 3 , and those for qualitative survival can be found in Table 4. As shown in Table 2, the RPA, SIR, and GPA grading systems had large discrepancies in patient numbers among groups. A total of $81.1 \%$ of patients were classified as RPA Class II and $76.1 \%$ as SIR Scores $4-7$, whereas only $1.7 \%$ were GPA Scores 3.5-4.0 and 5.3\% were GPA Score 3.0. The RPA, SIR, BSBM, and modified RPA grading systems showed statistically significant differences among subclasses ( $\mathrm{p}$ $<0.0001$ for all adjacent subclasses). Looking at the GPA system, however, one found that the difference between GPA Scores 3.5-4.0 and GPA Score 3.0 did not reach statistical significance $(\mathrm{p}=0.3750)$. Table 3 shows poor prognostic factors for overall survival that were verified. The final multivariate analysis model revealed 4 highly significant poor prognostic factors (initial KPS score $<70 \%$, primary lesion other than lung or breast, number of brain metastases; maximum diameter of the largest tumor $>25$ $\mathrm{mm}$; and active extracranial disease). On the contrary, only the SIR shows excellent statistical results for predicting preservation of neurological function, as shown in Table 4. Although the other grading systems did not yield statistically significant differences, the BSBM and the modified RPA appear to be better for predicting qualitative survival. 
TABLE 1: Demographic characteristics in 2445 patients with brain metastasis

\begin{tabular}{|c|c|}
\hline Characteristic & No. of Patients $(\%)^{*}$ \\
\hline median age in yrs (range) & $65(27-94)$ \\
\hline age $\geq 65$ & $1253(51.2)$ \\
\hline \multicolumn{2}{|l|}{ sex } \\
\hline male & $1469(60.0)$ \\
\hline female & $976(40.0)$ \\
\hline \multicolumn{2}{|l|}{ primary cancer site } \\
\hline lung & $1615(66.1)$ \\
\hline alimentary tract & $316(12.9)$ \\
\hline breast & $259(10.6)$ \\
\hline urogenital organs & $131(5.4)$ \\
\hline others & $124(5.1)$ \\
\hline \multicolumn{2}{|l|}{ disease status } \\
\hline controlled primary cancer & $713(29.2)$ \\
\hline no non-brain metastasis & $1548(63.3)$ \\
\hline \multicolumn{2}{|l|}{ KPS score } \\
\hline $100 \%$ & $1128(46.1)$ \\
\hline $90 \%$ & $385(15.7)$ \\
\hline $80 \%$ & $358(14.6)$ \\
\hline $70 \%$ & $246(10.0)$ \\
\hline $60 \%$ & $271(11.1)$ \\
\hline $50 \%$ & $57(2.3)$ \\
\hline \multicolumn{2}{|l|}{ no. of brain tumors (\%) } \\
\hline 1 & $662(27.1)$ \\
\hline $2-4$ & $825(33.7)$ \\
\hline $5-10$ & $543(22.2)$ \\
\hline $11-25$ & $325(13.3)$ \\
\hline$>25$ & $90(3.7)$ \\
\hline \multicolumn{2}{|l|}{ median tumor vol in $\mathrm{cm}^{3}$ (range) } \\
\hline cumulative & $4.50(0.02-100.00)$ \\
\hline max lesion & $2.80(0.01-72.50)$ \\
\hline \multicolumn{2}{|l|}{ neurological status } \\
\hline symptomatic & $1346(55.1)$ \\
\hline asymptomatic & $1099(44.9)$ \\
\hline prior surgery for brain metastases & $325(13.3)$ \\
\hline prior WBRT for brain metastases & $169(6.9)$ \\
\hline \multicolumn{2}{|c|}{$\begin{array}{l}\text { salvage procedures after initial GKS for } \\
\text { recurrent brain metastases (1031) }\end{array}$} \\
\hline 1 & $497(20.3)$ \\
\hline 2 & $273(11.2)$ \\
\hline 3 & $119(4.9)$ \\
\hline$\geq 4$ & $142(5.8)$ \\
\hline salvage WBRT & $95(3.9)$ \\
\hline
\end{tabular}

* Unless otherwise indicated.

\section{Discussion}

After Gaspar et al. ${ }^{3}$ developed an index, based on the original Radiation Therapy Oncolology Group's RPA, for predicting life expectancies of patients with brain metas-
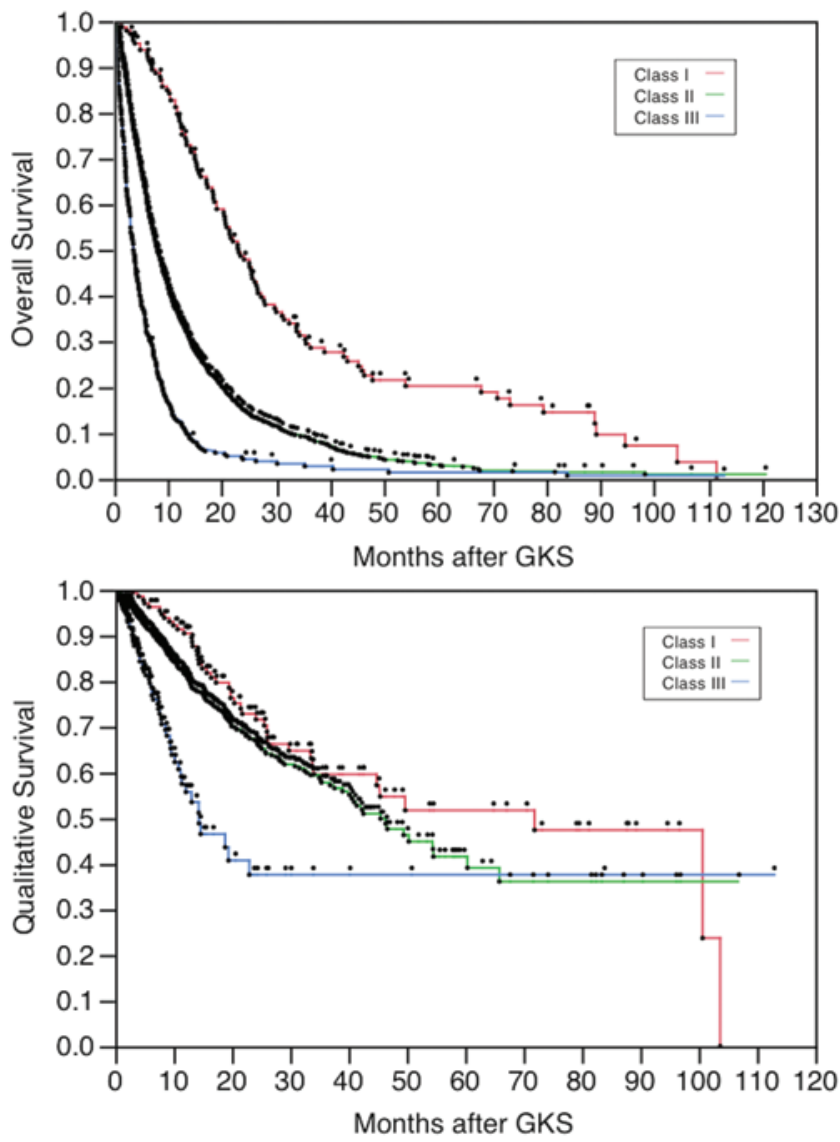

FIG. 1. Overall (upper) and qualitative (lower) survival curves according to the original RPA grading system. Red lines indicate RPA Class I; green lines, Class II; and blue lines, Class III. Black dots represent censored cases.

tasis, 4 additional grading systems were proposed: the SIR, ${ }^{14}$ the BSBM, ${ }^{7}$ the GPA, ${ }^{13}$ and the modified RPA. ${ }^{14}$ The SIR and the modified RPA were derived from a database of patients treated radiosurgically. ${ }^{14,16}$ We applied these 5 grading systems to nearly 2500 patients treated with GKS alone. As shown in Table 2, 4 of the 5 grading systems (specifically, the RPA, SIR, BSBM, and modified RPA) showed highly statistically significant differences when comparing pairs of adjacent classes $(p<0.001$ for all). In this series, in which patients in Japan were only treated with GKS, ${ }^{12,14}$ the difference between the GPA Score 3.5-4.0 and Score 3.0 groups did not reach statistical significance. These results were the same as those of Yamamoto's report on GKS-treated patients in Japan with quite similar backgrounds. In their report, the original RPA, as well as the SIR, BSBM, and modified RPA, were noted to be excellent grading systems for predicting overall survival. Unfortunately, all but the modified RPA have some faults. The BSBM does not incorporate brain disease status, that is, it does not include tumor number and tumor volume. On the other hand, although the SIR and GPA do include brain disease factors, their scoring seems rather complex for clinical use. There were large discrepancies in patient numbers among groups with the RPA, SIR, and GPA. Thus, we conclude that the modified 
T. Serizawa et al.
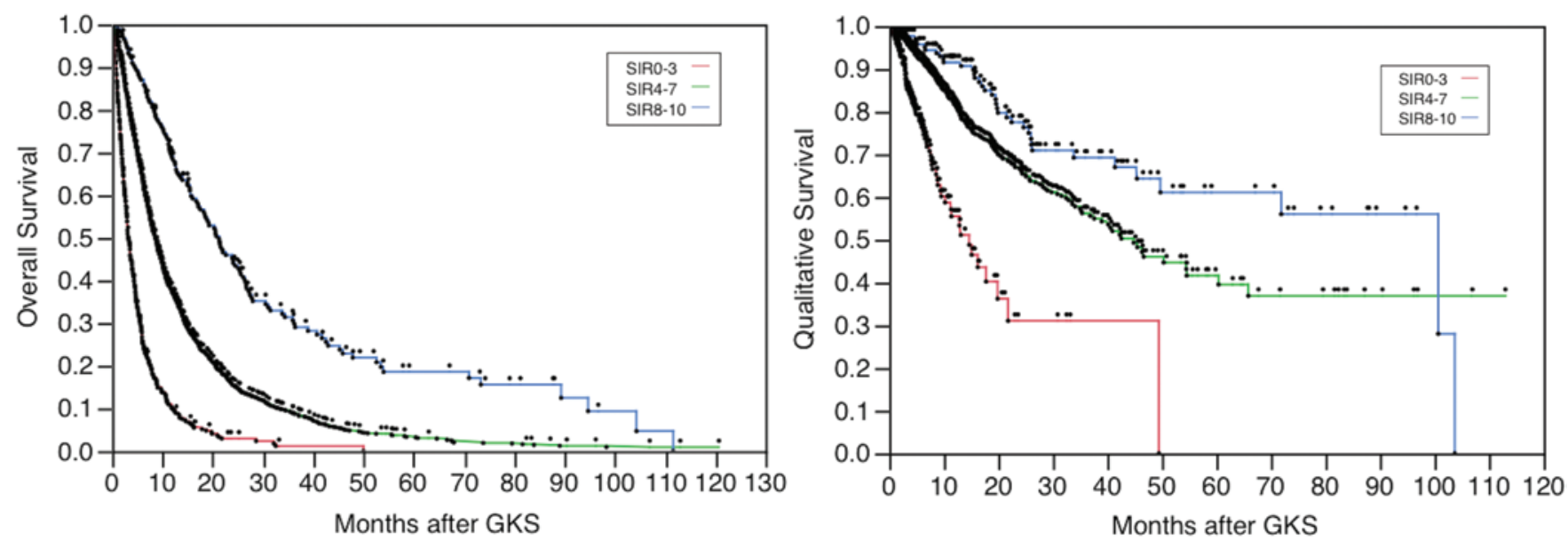

FIG. 2. Overall (left) and qualitative (right) survival curves according to the SIR grading system. Red lines indicate SIR Scores 0-3; green lines, SIR Scores 4-7; and blue lines, SIR Scores 8-10. Black dots represent censored cases.
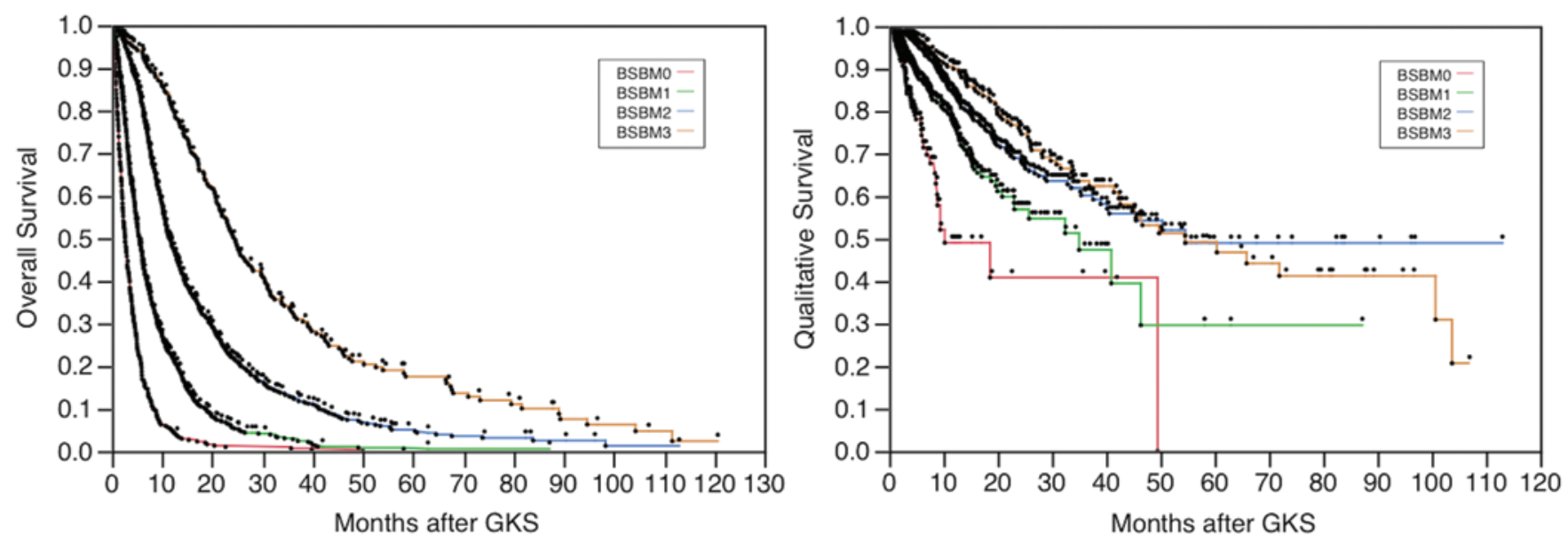

FIG. 3. Overall (left) and qualitative (right) survival curves according to the BSBM grading system. Red lines indicate BSBM Score 0; green lines, BSBM Score 1; blue lines, BSBM Score 2; and brown lines, BSBM Score 3. Black dots represent censored cases.
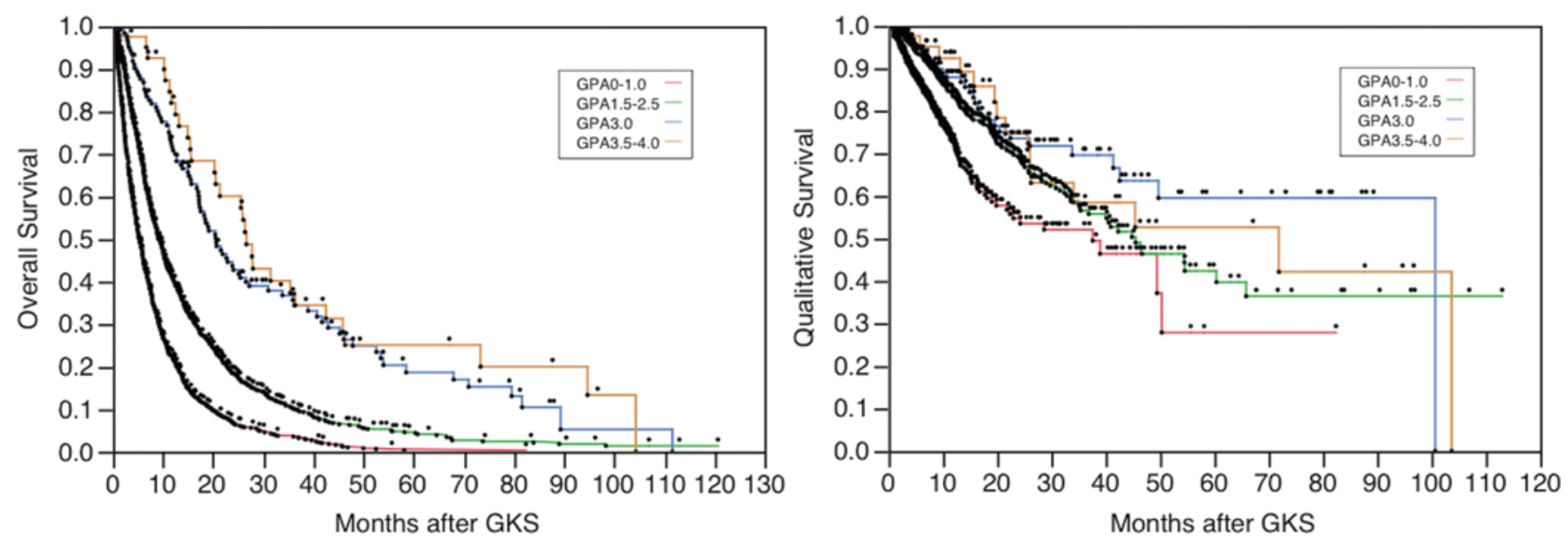

FIG. 4. Overall (left) and qualitative (right) survival curves according to the GPA grading system. Red lines indicate GPA Score 0-1.0; green lines, GPA Score 1.5-2.5; blue lines, GPA Score 3.0; and brown lines, GPA Score 3.5-4.0. Black dots represent censored cases. 

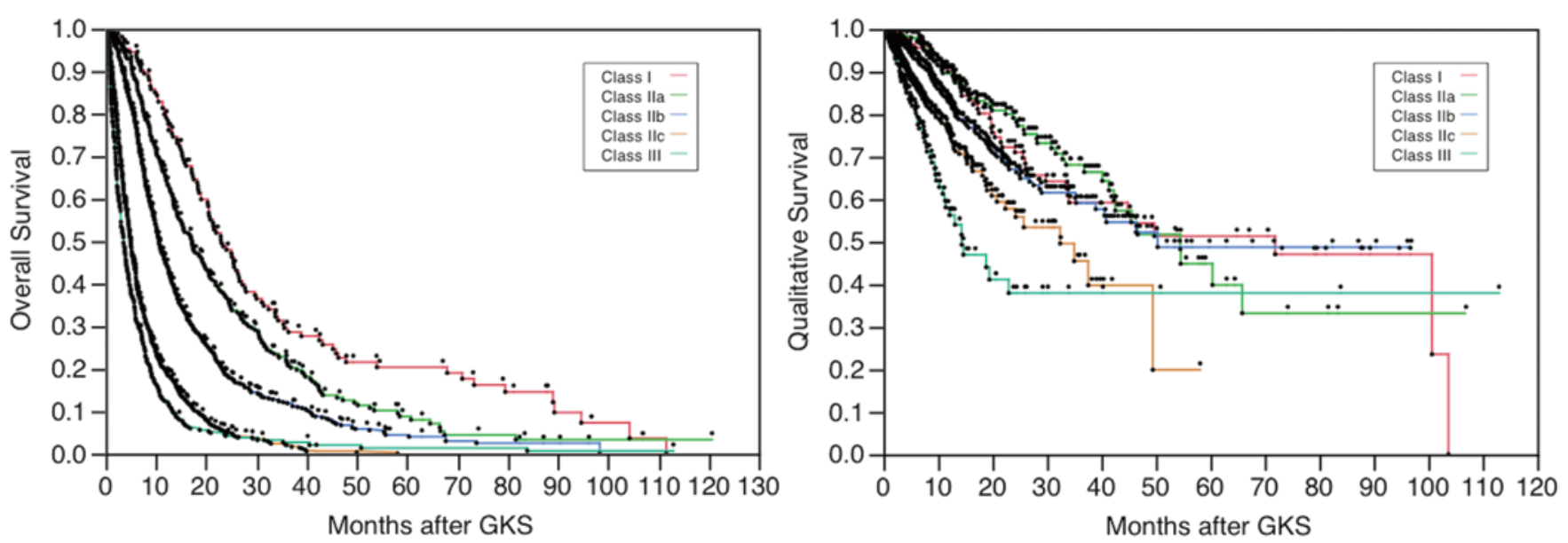

FIG. 5. Overall (left) and qualitative (right) survival curves according to the RPA modified by Yamamoto et al. Red lines indicate Class I; green lines, Class Ila; blue lines, Class IIb; brown lines, Class IIc; and dark green lines, Class III. Black dots represent censored cases.

RPA is well balanced and is the best grading system for the prediction of overall survival.

Preservation of function has been regarded as one of the ideal endpoints for evaluating treatment results for patients with brain metastases, even for those who undergo WBRT, surgical extirpation, SRT, or SRS. However, previous reports on grading systems for patients with brain metastases mention only overall survival and not preservation of function. We have followed up on patients with meticulous neurological examinations and enhanced MR imaging every 2-3 months, with special attention given to changes in performance status due to brain disease. We use "qualitative survival" to mean preservation of neurological function (equivalent to a KPS score of $\geq 70 \%$ ), de-

TABLE 2: Statistical results of overall survivals according to the 5 major grading systems*

\begin{tabular}{|c|c|c|c|c|c|c|c|}
\hline Grading & No. of Patients & MST (mos) & $95 \% \mathrm{Cl}$ & Comparison & $\mathrm{HR}$ & $95 \% \mathrm{Cl}$ & $\mathrm{p}$ Value \\
\hline \multicolumn{8}{|l|}{ RTOG RPA } \\
\hline Class I & 146 & 23.1 & $20.3-26.6$ & & & & \\
\hline Class II & 1983 & 8.0 & $7.6-8.6$ & vs I & 2.381 & $1.969-2.912$ & $<0.0001$ \\
\hline Class III & 316 & 3.6 & $3.0-4.2$ & vs II & 2.018 & $1.778-2.282$ & $<0.0001$ \\
\hline \multicolumn{8}{|l|}{ SIR } \\
\hline Score 8-10 & 404 & 21.3 & $17.1-25.4$ & & & & \\
\hline Score 4-7 & 1860 & 8.6 & $8.0-9.0$ & vs $8-10$ & 2.192 & $1.841-2.634$ & $<0.0001$ \\
\hline Score 0-3 & 181 & 3.2 & $2.9-3.7$ & vs $4-7$ & 2.544 & $2.789-2.853$ & $<0.0001$ \\
\hline \multicolumn{8}{|l|}{ BSBM } \\
\hline Score 3 & 262 & 24.6 & $21.6-27.8$ & & & & \\
\hline Score 2 & 857 & 11.6 & $10.7-12.6$ & vs 3 & 1.903 & $1.622-2.243$ & $<0.0001$ \\
\hline Score 1 & 981 & 5.4 & $5.0-6.0$ & vs 2 & 2.218 & $2.003-2.456$ & $<0.0001$ \\
\hline Score 0 & 345 & 2.6 & $2.2-3.0$ & vs 1 & 2.264 & $1.990-2.570$ & $<0.0001$ \\
\hline \multicolumn{8}{|l|}{ GPA } \\
\hline Score 3.5-4.0 & 41 & 26.7 & $20.3-36.4$ & & & & \\
\hline Score 3.0 & 129 & 20.6 & $17.2-25.4$ & vs $3.5-4.0$ & 1.202 & $0.8056-1.846$ & 0.3750 \\
\hline Score $1.5-2.5$ & 1242 & 9.2 & $8.7-10.1$ & vs 3.0 & 2.078 & $1.686-2.600$ & $<0.0001$ \\
\hline Score $0-1.0$ & 1033 & 5.3 & $4.9-5.9$ & vs $1.5-2.5$ & 1.738 & $1.588-1.901$ & $<0.0001$ \\
\hline \multicolumn{8}{|c|}{ Yamamoto's modified RPA } \\
\hline Class I & 146 & 23.1 & $20.4-26.6$ & & & & \\
\hline Class Ila & 304 & 17.2 & $14.3-19.8$ & vs I & 1.367 & $1.093-1.721$ & 0.0060 \\
\hline Class IIb & 696 & 10.7 & $10.0-11.6$ & vs Ila & 1.475 & $1.271-1.717$ & $<0.0001$ \\
\hline Class IIc & 983 & 5.0 & $4.6-5.3$ & vs Ilb & 2.167 & $1.947-2.414$ & $<0.0001$ \\
\hline Class III & 316 & 3.6 & $3.0-4.1$ & vs IIc & 2.656 & $2.304-3.056$ & 0.0030 \\
\hline
\end{tabular}

* MST = median survival time; $\mathrm{RTOG}=$ Radiation Therapy Oncology Group. 
TABLE 3: Prognostic values for overall survival

\begin{tabular}{|c|c|c|c|c|c|}
\hline \multirow[b]{2}{*}{ Variable } & \multirow[b]{2}{*}{ High-Risk Group } & \multicolumn{2}{|c|}{ Univariate Analysis } & \multicolumn{2}{|c|}{ Multivariate Analysis } \\
\hline & & p Value & $\mathrm{HR}(95 \% \mathrm{Cl})$ & p Value & $\operatorname{HR}(95 \% \mathrm{Cl})$ \\
\hline age & $\geq 65 \mathrm{yrs}$ & 0.0108 & $1.118(1.061-1.217)$ & & \\
\hline initial KPS score & $<70 \%$ & $<0.0001$ & $2.807(2.441-3.243)$ & $<0.0001$ & $2.124(1.867-2.410)$ \\
\hline primary lesion & not lung or breast & $<0.0001$ & $1.510(1.365-1.668)$ & $<0.0001$ & $1.392(1.253-1.545)$ \\
\hline MR findings of CSF dissemination & positive & 0.0002 & $1.262(1.110-1.428)$ & & \\
\hline craniotomy & no & 0.5478 & $1.039(0.918-1.181)$ & & \\
\hline chemotherapy & no & 0.6958 & $1.017(0.934-1.108)$ & & \\
\hline diagnostic lag & synchronous & 0.3903 & $1.040(0.951-1.136)$ & & \\
\hline prior WBRT & yes & 0.0421 & $1.196(1.007-1.410)$ & & \\
\hline
\end{tabular}

fined as no impairment due to any type of brain disease, that is, tumor recurrence, radiation injury, cerebral dissemination, CSF dissemination, and other brain diseases. In this paper, we focused on which grading system is the best for predicting preservation of neurological function. From the standpoint of overall survival, 4 of the grading systems - all but the GPA-were excellent. Considering both overall and qualitative survival, however, the SIR appears to be the best grading system. These results allow us to conclude that patients with SIR scores of 8-10 are excellent candidates for GKS. Those with SIR scores of 4-7 are also good candidates, based on overall as well

TABLE 4: Statistical results of qualitative survivals according to the 5 major grading systems

\begin{tabular}{|c|c|c|c|c|c|c|c|}
\hline Grading & No. of Patients & $6 \operatorname{Mos}(\%)$ & $1 \mathrm{Yr}(\%)$ & Comparison & $\mathrm{HR}$ & $95 \% \mathrm{Cl}$ & p Value \\
\hline Class I & 146 & 96.2 & 90.5 & & & & \\
\hline Class III & 313 & 79.6 & 57.6 & vs II & 2.344 & $1.771-3.052$ & $<0.0001$ \\
\hline \multicolumn{8}{|l|}{ SIR } \\
\hline Score 0-3 & 180 & 76.4 & 55.5 & vs $4-7$ & 3.040 & $2.346-3.893$ & $<0.0001$ \\
\hline \multicolumn{8}{|l|}{ BSBM } \\
\hline Score 3 & 262 & 96.3 & 89.9 & & & & \\
\hline Score 2 & 855 & 94.5 & 84.1 & vs 3 & 1.144 & $0.871-1.516$ & 0.3360 \\
\hline Score 1 & 979 & 87.5 & 76.6 & vs 2 & 1.731 & $1.378-2.173$ & $<0.0001$ \\
\hline \multicolumn{8}{|l|}{ GPA } \\
\hline Score 3.0 & 128 & 93.3 & 83.3 & vs $3.5-4.0$ & 0.815 & $0.115-1.558$ & 0.5243 \\
\hline Score 1.5-2.5 & 1241 & 93.7 & 87.9 & vs 3.0 & 1.383 & $0.957-2.073$ & 0.0859 \\
\hline Score 0-1.0 & 1029 & 95.1 & 92.3 & vs $1.5-2.5$ & 1.981 & $1.199-3.534$ & 0.0063 \\
\hline \multicolumn{8}{|c|}{ Yamamoto's modified RPA } \\
\hline Class I & 146 & 95.6 & 89.9 & & & & \\
\hline Class Ila & 304 & 97.9 & 89.6 & vs I & 1.175 & $0.795-1.723$ & 0.4138 \\
\hline Class IIb & 693 & 93.1 & 83.0 & vs Ila & 1.360 & $1.009-1.853$ & 0.0435 \\
\hline Class Ilc & 783 & 86.7 & 75.8 & vs Ilb & 1.678 & $1.318-2.139$ & $<0.0001$ \\
\hline Class III & 313 & 80.2 & 58.0 & vs Ilc & 1.561 & $1.149-2.096$ & 0.0048 \\
\hline
\end{tabular}




\section{Grading systems for brain metastases treated radiosurgically}

as qualitative survival, but SRS is contraindicated in patients with SIR Scores 0-3. Nevertheless, SIR has one major fault. This scoring system is extremely complex for clinical use, because it has 5 items with 3 different scores and includes 2 brain disease factors: the number of brain tumors $(1,2$, and $>2$ tumors) and total brain tumor volume $\left(<5 \mathrm{~cm}^{3}, 5-13 \mathrm{~cm}^{3}\right.$, and $\left.>13 \mathrm{~cm}^{3}\right)$. Indeed, brain disease factors, including tumor number, tumor location, maximum tumor number, total tumor number, and CSF dissemination, are considered crucial for choosing among treatment modalities including WBRT, craniotomy, SRT, SRS, and best supportive care. Thus, a new grading system for predicting both overall survival and qualitative survival and for selecting the best treatment for patients with brain metastasis is needed. The modified RPA, proposed by Yamamoto et al. ${ }^{16}$ has the potential to resolve these problems. Its only disadvantage is the lack of a significant difference between Classes I and IIa, both of which contain relatively small numbers of patients, such that combining Classes I and IIa may be an option. However, a new grading system focused on qualitative survival, as well as on overall survival, is necessary. This would allow poor prognostic factors for qualitative survival to be analyzed in our large patient series treated with GKS.

\section{Conclusions}

Among the 5 major grading systems, four-the RPA, SIR, BSBM, and modified RPA-yielded highly statistically significant differences in our 2500 cases treated with GKS. For predicting preservation of function, which we consider to be the most important evaluation endpoint, the SIR was apparently the best, while the BSBM and the modified RPA also produced good statistical results. Unfortunately, the SIR scoring is overly complex for clinical use. Thus, a new simple grading system is necessary for the prediction of qualitative as well as overall survival and for selecting an optimal brain metastasis patient cohort for SRS.

\section{Disclosure}

The authors report no conflict of interest concerning the materials or methods used in this study or the findings specified in this paper.

Author contributions to the study and manuscript preparation include the following. Conception and design: Serizawa. Acquisition of data: Serizawa, Higuchi, Nagano. Analysis and interpretation of data: Serizawa, Higuchi, Nagano. Drafting the article: Serizawa. Critically revising the article: all authors. Reviewed submitted version of manuscript: all authors. Approved the final version of the manuscript on behalf of all authors: Serizawa. Statistical analysis: Serizawa, Higuchi. Study supervision: Hirai, Ono, Saeki, Miyakawa.

\section{References}

1. Aoyama H, Shirato H, Tago M, Nakagawa K, Toyoda T, Hatano $\mathrm{K}$, et al: Stereotactic radiosurgery plus whole-brain radiation therapy vs stereotactic radiosurgery alone for treatment of brain metastases: a randomized controlled trial. JAMA 295:24832491, 2006
2. Cox DR: Regression models and life tables. J R Stat Soc B 34: 187-220, 1972

3. Gaspar L, Scott C, Rotman M, Asbell S, Phillips T, Wasserman $\mathrm{T}$, et al: Recursive partitioning analysis (RPA) of prognostic factors in three Radiation Therapy Oncology Group (RTOG) brain metastases trials. Int J Radiat Oncol Biol Phys 37:745751,1997

4. Higuchi Y, Serizawa T, Nagano O, Matsuda S, Ono J, Sato M, et al: Three-staged stereotactic radiotherapy without whole brain irradiation for large metastatic brain tumors. Int J Radiat Oncol Biol Phys 74:1543-1548, 2009

5. Kaplan EL, Meier P: Nonparametric estimation from incomplete observations. J Am Stat Assoc 53:457-481, 1958

6. Karnofsky DA, Burchenal JH: The clinical evaluation of chemotherapeutic agents in cancer, in MacLeod CM (ed): Evaluation of Chemotherapeutic Agents. New York: Columbia University Press, 1949, pp 191-205

7. Lorenzoni J, Devriendt D, Massager N, David P, Ruíz S, Vanderlinden B, et al: Radiosurgery for treatment of brain metastases: estimation of patient eligibility using three stratification systems. Int J Radiat Oncol Biol Phys 60:218-224, 2004

8. Serizawa T, Higuchi Y, Ono J, Matsuda S, Nagano O, Iwadate Y, et al: Gamma Knife surgery for metastatic brain tumors without prophylactic whole-brain radiotherapy: results in 1000 consecutive cases. J Neurosurg 105 Suppl:86-90, 2006

9. Serizawa T, Iuchi T, Ono J, Saeki N, Osato K, Odaki M, et al: Gamma knife treatment for multiple metastatic brain tumors compared with whole-brain radiation therapy. J Neurosurg 93 (Suppl 3):32-36, 2000

10. Serizawa T, Ono J, Iichi T, Matsuda S, Sato M, Odaki M, et al: Gamma knife radiosurgery for metastatic brain tumors from lung cancer: a comparison between small cell and non-small cell carcinoma. J Neurosurg 97 (Suppl 5):484-488, 2002

11. Serizawa T, Saeki N, Higuchi Y, Ono J, Iuchi T, Nagano O, et al: Gamma knife surgery for brain metastases: indications for and limitations of a local treatment protocol. Acta Neurochir (Wien) 147:721-726, 2005

12. Serizawa T, Yamamoto M, Nagano O, Higuchi Y, Matsuda S, Ono J, et al: Gamma Knife surgery for metastatic brain tumors. A 2-institute study in Japan. J Neurosurg 109 Suppl:118-121, 2008

13. Sperduto PW, Berkey B, Gaspar LE, Mehta M, Curran W: A new prognostic index and comparison to three other indices for patients with brain metastases: an analysis of 1,960 patients in the RTOG database. Int J Radiat Oncol Biol Phys 70:510 514,2008

14. Weltman E, Salvajoli JV, Brandt RA, de Morais Hanriot R, Prisco FE, Cruz JC, et al: Radiosurgery for brain metastases: a score index for predicting prognosis. Int J Radiat Oncol Biol Phys 46:1155-1161, 2000

15. Yamamoto M, Ide M, Nishio S, Urakawa Y: Gamma Knife radiosurgery for numerous brain metastases: is this a safe treatment? Int J Radiat Oncol Biol Phys 53:1279-1283, 2002

16. Yamamoto M, Sato Y, Serizawa T, Kawabe T, Higuchi Y, Nagano O, et al: Sub-classification of recursive partitioning analysis class II patients with brain metastases treated radiosurgically. Int J Radiat Oncol Biol Phys 83:1399-1405, 2012

Manuscript submitted April 9, 2012.

Accepted August 1, 2012.

This paper was presented at the 16th International Leksell Gamma Knife Society Meeting, Sydney, Australia, March 25-29, 2012.

Please include this information when citing this paper: DOI: 10.3171/2012.8.GKS12710.

Address correspondence to: Toru Serizawa, M.D., Tokyo Gamma Unit Center, Tsukiji Neurologic Clinic, 1-9-9 Tsukiji, Chuoku, Tokyo 104-0045. Japan. email: gamma-knife.serizawa@nifty.com. 